\title{
ESTUDIO BIOANTROPOLÓGICO DEL SURCO PALMAR TRANSVERSO EN LOS MAYAS YUCATECOS
}

\author{
Carlos Serrano \\ Julieta ArÉchiga \\ Instituto de Investigaciones \\ Antropológicas, UNAM.
}

\section{Introducción}

Los dermatoglifos (derma, dermatos, piel; glyphoo, esculpir), son las figuras formadas por los relieves dérmicos, en las regiones palmar y plantar del cuerpo humano, con una base genética y un significado evolutivo propios de nuestra especie.

Sus manifestaciones en las poblaciones humanas son, sin embargo, variables, y este hecho confiere a los dermatoglifos una relevancia antropológica de primer orden en virtud de la información que pueden proporcionar acerca de la filiación biológica de los diversos grupos humanos.

Entre los rasgos morfológicos analizables en las impresiones dígitopalmares, que se obtienen con esta finalidad, se encuentra un carácter estrechamente relacionado con los dermatoglifos y que ha sido objeto de numerosos estudios particulares. Se trata del surco palmar transverso, que es una variante de la configuración de los surcos de flexión de la palma de la mano. El establecimiento de su frecuencia en un grupo humano dado, teniendo en cuenta la distribución geográfica del carácter, puede aportar un conocimiento valioso para el estudio antropológico del grupo y su ubicación en la gama de variación somática de las poblaciones humanas.

Sin embargo, los datos publicados hasta ahora sobre tal carácter se refieren principalmente a poblaciones del Viejo Mundo, siendo notoria la escasez de información sobre los grupos indígenas americanos. Para suplir en parte esta falta de datos nos proponemos, en este trabajo, examinar la manifestación del surco palmar transverso en un grupo de ascendencia biológica prehispánica, los mayas yucatecos. Otros as- 
pectos dermatoglíficos en el mismo grupo serán abordados posteriormente.

Este estudio se inscribe, por otra parte, en una investigación más amplia que persigue, a través del análisis de marcadores genéticos y datos antropométricos, mejorar el conocimiento antropológico-físico de las poblaciones indígenas actuales del sureste de México, clasificadas dentro del trono lingüístico mayance.

\section{El material estudiado}

Los materiales examinados fueron recogidos en diversas temporadas de trabajo de campo, a partir de 1971, en varias comunidades indígenas de Yucatán y Quintana Roo, como parte de las investigaciones

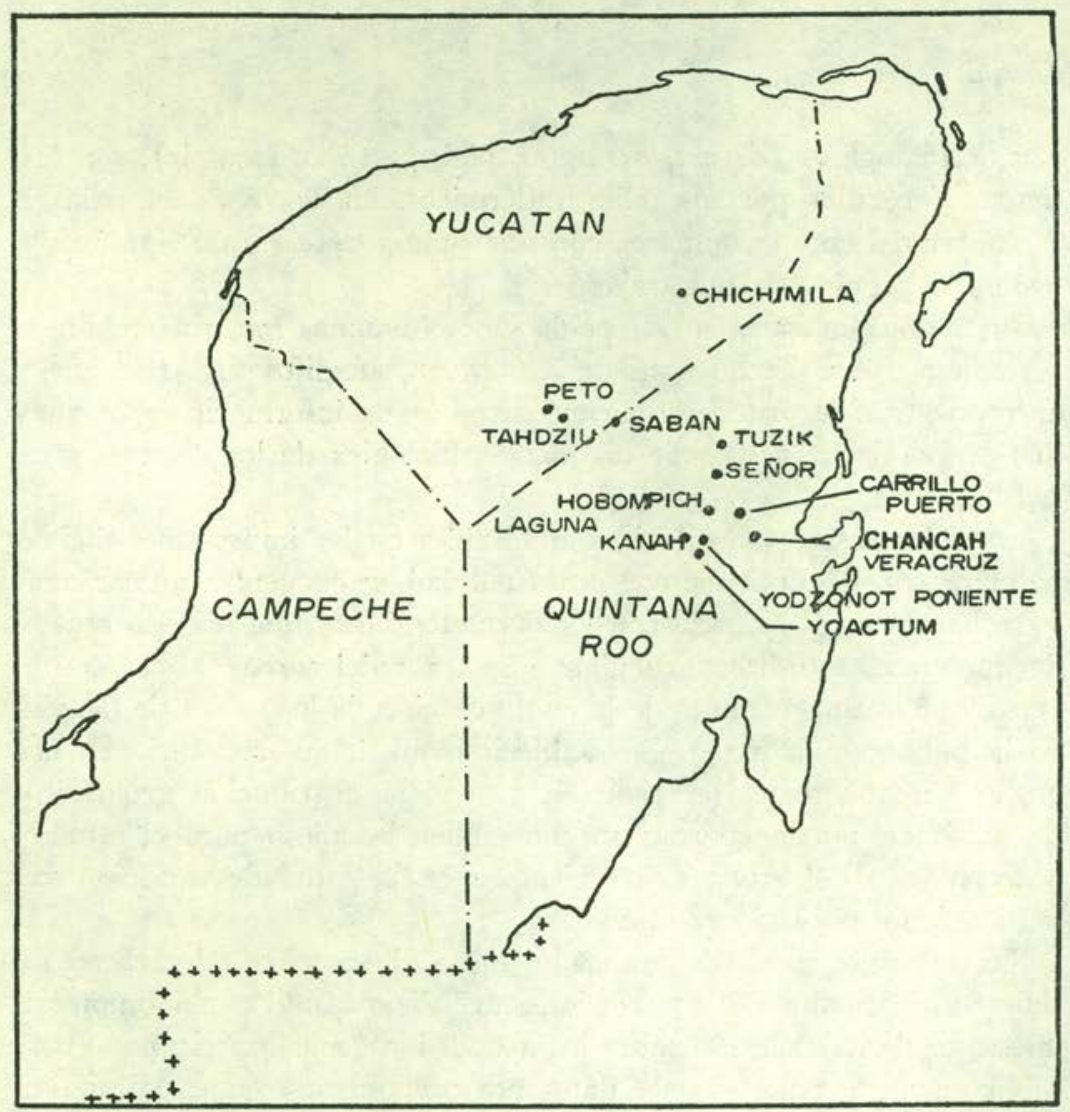

FIG. 1- LOCALIZACION DE LOS SITIOS DE PROCEDENCIA DE LOS MATERIALES ESTUDIADOS. 


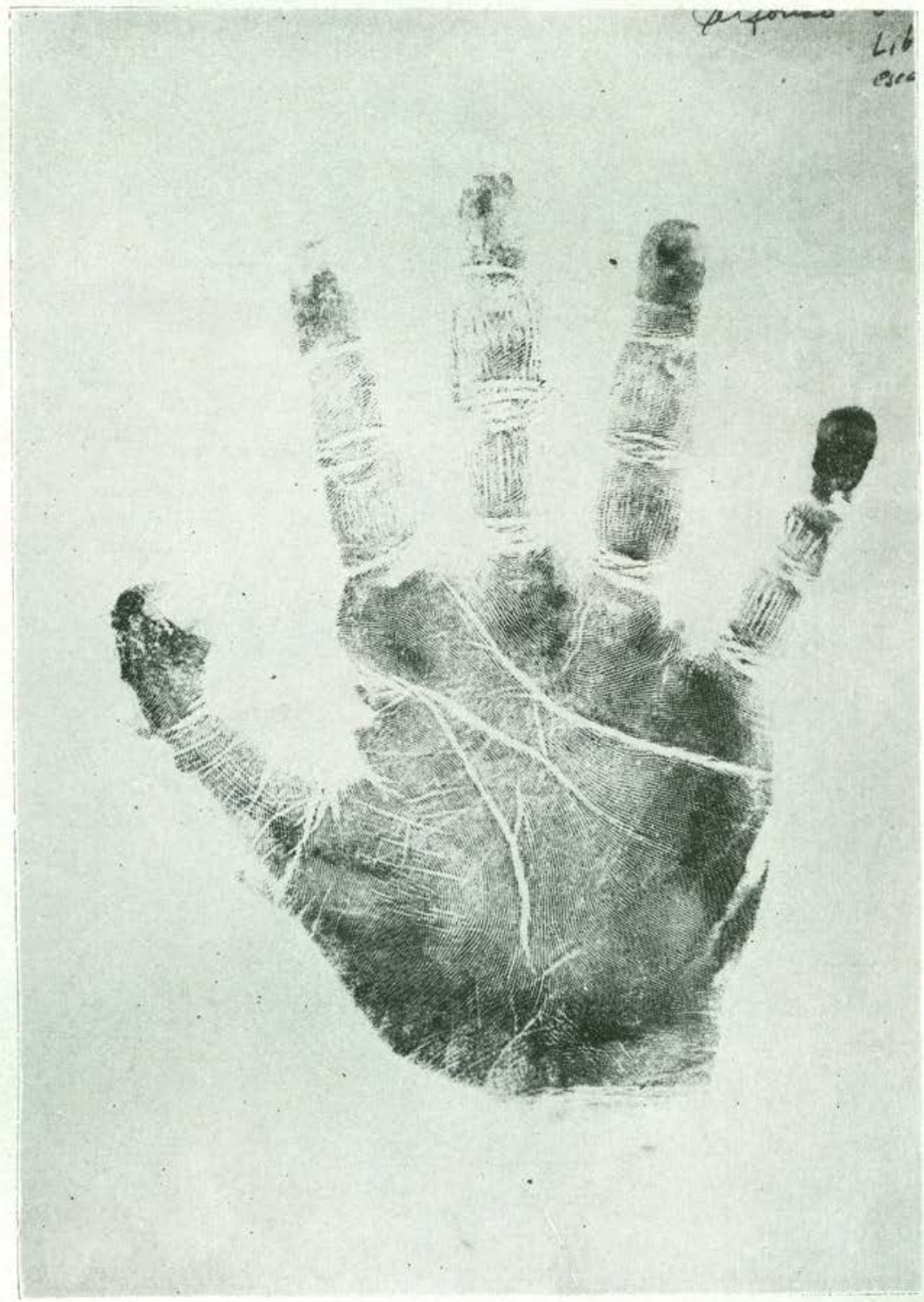

LÁm. I. Configuración normal de los surcos de flexión de la palma de la mano.

Estudios de Cultura Maya. Vol. XII, 1979

Instituto de Investigaciones Filológicas/

Centro de Estudios Mayas, UNAM

http://www.iifilologicas.unam.mx/estculmaya/ 


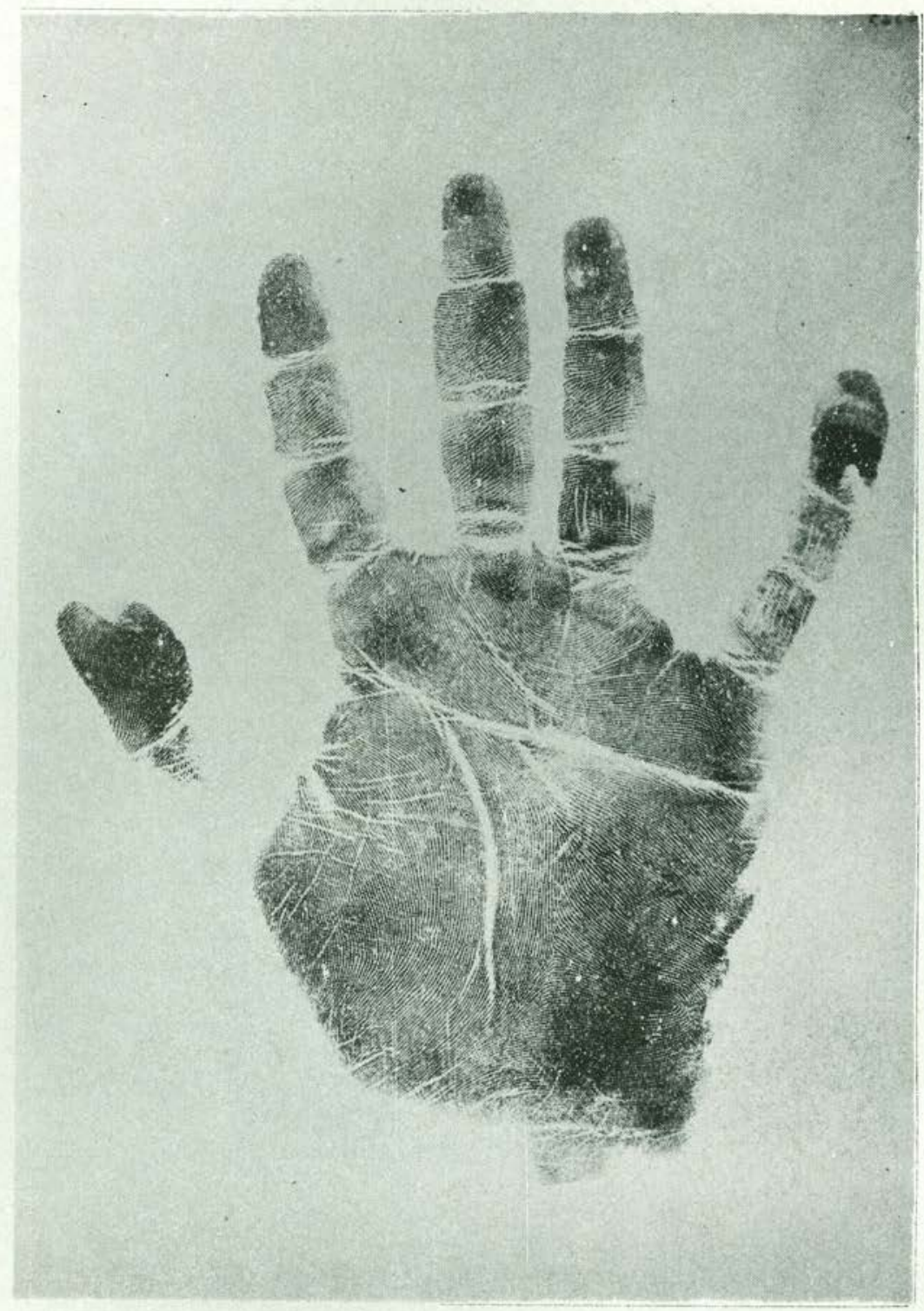

LÁm. II. Un caso de surco palmar transverso: un pliegue de flexión recorre transversalmente la palma del borde radial al borde cubital, aunque persisten fragmentos residuales de los pliegues de flexión de los tres y de los cuatro últimos dedos.

Estudios de Cultura Maya. Vol. XII, 1979

Instituto de Investigaciones Filológicas/

Centro de Estudios Mayas, UNAM

http://www.iifilologicas.unam.mx/estculmaya/ 
en Antropología física programadas en el Centro de Estudios Mayas de la UNAM.

Los sitios visitados, en los cuales se obtuvieron los materiales de estudio, están situados hacia la parte oriental de la Península de Yucatán (fig. 1); en esta región como es sabido, las comunidades mayas han mantenido su patrimonio cultural (Villa Rojas, 1945) y el mestizaje biológico, cuando se ha producido, apenas es discernible.

Dado el propósito de este trabajo, la muestra de impresiones dígitopalmares que se estudió, fue depurada eliminando a los sujetos cuyas familias se habían avecindado recientemente en las comunidades visitadas y cuya ascendencia generalmente era mestiza, lo cual podía apreciarse fácilmente a través de algunos rasgos europeoides del fenotipo. Por otra parte, la gran mayoría de los sujetos estudiados tenía ambos apellidos mayas, lo cual puede considerarse un buen indicador de ascendencia indígena, aunque un número reducido de sujetos con un solo patronímico maya también se incluyó en la muestra. Por el contrario, los escasos individuos cuyos apellidos eran de origen español fueron excluidos del estudio.

Las impresiones palmares examinadas pertenecen a escolares de ambos sexos, cuyas edades fluctuaban entre 7 y 14 años. El total de sujetos considerados fue de 552 individuos de sexo masculino y 409 del femenino; su número y lugar de procedencia se consignan en el Cuadro 1.

\section{Definición y nomenclaturas para el estudio del surco palmar transverso}

La superficie de la palma de la mano está recorrida normalmente por tres surcos o pliegues de flexión (lám. 1): el del pulgar, el de los cuatro últimos dedos y el de los tres últimos dedos, que en la figura 2 pueden identificarse con los números 1,2 y 3 , respectivamente. El pliegue 1 presenta variaciones generalmente ligeras; los pliegues 2 y 3 , en cambio, pueden presentar una configuración muy diferente de la considerada como normal. En efecto, cuando estos pliegues son reemplazados por un solo surco que cruza la palma del borde radial al cubital, persistan o no porciones vestigiales de dichos surcos, se configura el llamado surco palmar transverso (lám. 2).

Este rasgo morfológico, sin embargo, puede presentar variaciones, de tal manera que pueden identificarse casos transicionales entre la disposición normal de los surcos de flexión y el surco palmar transverso "completo" o "típico". 
ESTUDIO DEL SURCO PALMAR TRANSVERSO EN POBLACION MAYA YUCATECA. NUMERO Y PROCEDENCIA DE; LOS INDIVIDUOS EXAMINADOS

\begin{tabular}{lrr}
\hline & Hombres & Mujeres \\
\hline & & \\
YUCATAN: & 64 & 51 \\
Chichimilá & 124 & 99 \\
Peto $^{1}$ & 45 & 37 \\
Tahdziu & 233 & 187 \\
\cline { 2 - 3 } & & \\
QUINTANA ROO: & 40 & \\
Sabán & 18 & 45 \\
Laguna Kanah & 12 & 11 \\
Chancah Veracruz & 57 & 19 \\
Señor & 23 & 17 \\
Tusik & 14 & 29 \\
Yoactum & 16 & 8 \\
Hobompich & 11 & 14 \\
Yodzonot Pte. & 128 & 79 \\
Carrillo Puerto & & \\
& 319 & 222 \\
\hline
\end{tabular}

1 Los sujetos estudiados provienen de un barrio indígena de esta localidad.

2 Esta serie se obtuvo en un internado indígena; los sujetos estudiados son originarios de diversas comunidades mayas del Municipio de Carrillo Puerto.

Este último ha sido objeto de estudio desde el punto de vista de la Genética Clínica, por su asociación con diversas aberraciones y anomalías congénitas, que le otorgan un valor diagnóstico en la práctica médica (Holt, 1968; Schaumann y Alter, 1975). Sin embargo, su presencia se detecta también en sujetos normales, y en este sentido, su frecuencia en poblaciones humanas puede ser analizada antropológicamente.

Bajo tal enfoque, no obstante, las diversas manifestaciones morfológicas del surco palmar transverso representan también motivo de estudio. Así, las clasificaciones propuestas para abarcar la variación entre la configuración normal y el surco transverso típico consideran un cierto número de tipos (Brander, 1940; Khérumian, et al., 1955; Weninger y Navratil, 1957; Almeida Santos, 1960; Kimura, 1968; Lestrange, 1969\}. 


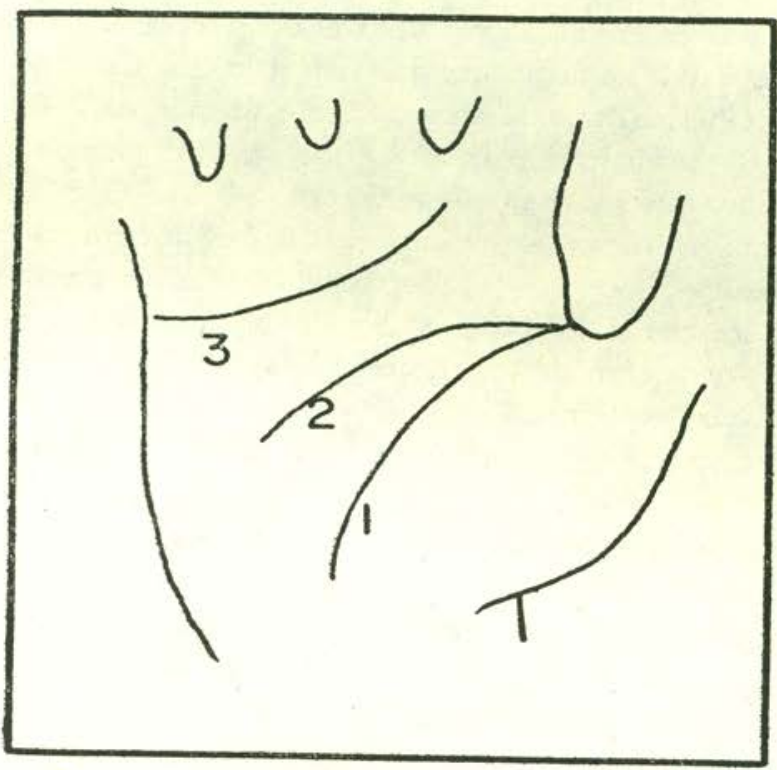

FIG. 2- CONFIGURACION NORMAL DE LOS SURCOS DE FLEXION DE LA PALMA DE LA MANO: 1) DEL PULGAR, 2) DE LOS CUATRO ULTIMOS DEDOS. 3) DE LOS TRES ULTIMOS

En estas clasificaciones la definición del pliegue transverso típico es prácticamente la misma y las configuraciones que se consideran relacionadas a este último son muy parecidas. De hecho, al decir de Lestrange (1969, p. 252), se trata más bien de nomenclaturas que de clasificaciones. Sin embargo, la clasificación propuesta por dicha investigadora tiene la ventaja de estar basada en criterios bien definidos, cuya aplicación resulta sencilla y práctica, lo cual permite al mismo tiempo una precisión aceptable para el registro del carácter. Por estos motivos se le ha adoptado para el presente trabajo.

En dicha clasificación, los pliegues de flexión que atraviesan la palma del borde radial al borde cubital, se agrupan en dos categorías, según sus características (fig. 3) : pliegues transversos y pliegues transversales.

Los pliegues transversos reúnen las tres características siguientes:

a) el pliegue es rectilíneo,

b) sus puntos terminales ocupan los sitios de origen de los pliegues

2 y 3 en las palmas normales,

c) los pliegues 2 y 3 participan en su formación. 
El tipo I corresponde al pliegue transverso típico (P.t.); reemplaza a los pliegues 2 y 3 de los cuales subsisten a lo más dos fragmentos cortos libres (Ptf). El tipo II corresponde a un pliegue 2 (o excepcionalmente 3 ) normal; el pliegue 3 (o 2 ) es reemplazado por un pliegue rectilíneo que se arroja en el primero $(3 \rightarrow 2,2 \rightarrow 3)$; se pueden presentar también fragmentos residuales del pliegue cuyo trayecto se modificó $(3 \rightarrow 2+\mathrm{f}, 2 \rightarrow 3+\mathrm{f})$. Se clasifican también como tipo II los casos en los cuales los pliegues 2 y 3 son normales pero se encuentran unidos por un puente, de tal manera que se configura un pliegue transverso rectilíneo.

El tipo II corresponde al surco palmar transverso transicional o incompleto de otros autores.

En los pliegues transversales (tipos III y IV) uno solo de los pliegues, 2 o 3, atraviesa la palma, presentando el otro un trayecto normal, por lo cual la característica $c$ de los pliegues transversos no se cumple, en tanto que $a$ y $b$ pueden estar o no presentes.

En el tipo III, el pliegue transversal está constituido únicamente por el pliegue 3, que puede ser sinuoso (PtS) o rectilíneo (PtR), en tanto que el tipo IV se forma sólo con el pliegue $2(2 t)$, que presenta una dirección muy oblícua, o raramente con el 3 (3t) que aun siendo transversal y rectilíneo describe un trayecto independiente de los dos pliegues restantes.

Las formas atípicas (tipo V) agrupan las configuraciones en las cuales los trayectos de los tres pliegues principales puede ser muy diferentes de la normal, sin presentar un elemento que atraviesa la palma del borde radial al ulnar.

\section{Distribución de los difentes tipos de pliegues palmares en función del sexo}

a) En el cuadro 2 se presenta la distribución de las diferentes configuraciones de los pliegues palmares en cada mano, en la serie mas culina.

En el Cuadro 3 se han reunido las 2 categorías de pliegues transver$\operatorname{sos}(I+I I)$ y de pliegues transversales $(I I I+I V)$, considerando que corresponden a etiologías diferentes, tal como ha sido mostrado por Lestrange (1969). El tipo V (formas atípicas), se ha reunido con el tipo 0 (configuración normal), bajo el criterio de que ambos tipos no presentan el elemento de transversalidad de un surco de flexión, del borde radial al borde cubital de la palma. 


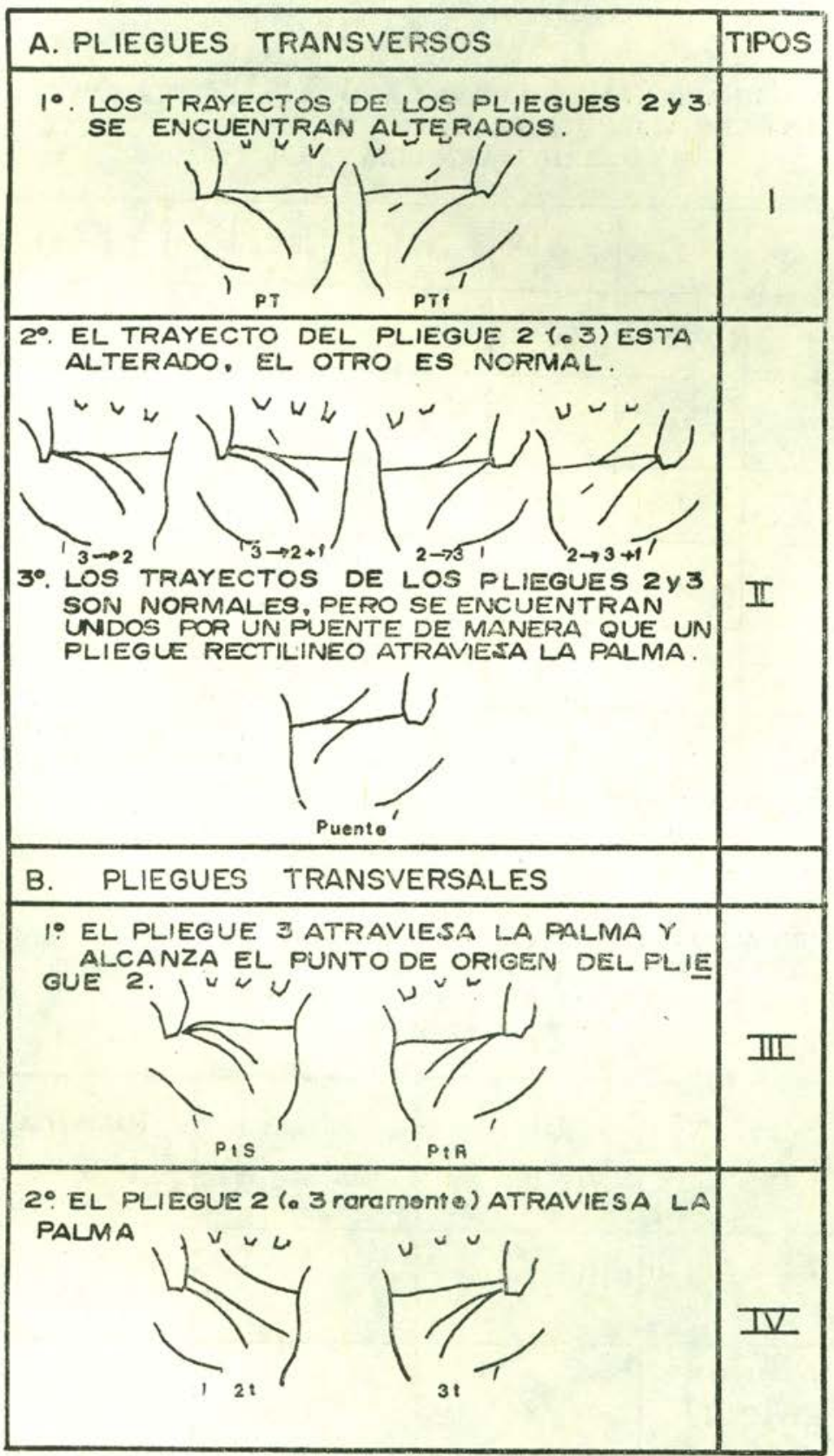

FIG. 3 - PLIEGUES PALMARES TRANSVERSOS $Y$ TRANSVERSALES, SEGUN LA CLASIFICACION DE LESTRANGE, 1969 . 
CUADRo 2

COMBINACIONES EN AMBAS MANOS DE LOS DIFERENTES TIPOS DE PLIEGUES PALMARES EN MAYAS YUCATECOS. SERIE MASCULINA $(\mathrm{n}=552)$

\begin{tabular}{|c|c|c|c|c|c|c|}
\hline & 0 & $v$ & IV & III & $\|$ & 1 \\
\hline 1 & 12 & 0 & 0 & 0 & 4 & 4 \\
\hline 18 & 28 & 0 & 0 & 0 & 7 & \\
\hline ISI & 1 & 0 & 0 & 0 & & \\
\hline iv & 1 & 0 & 1 & & & \\
\hline$v$ & 7 & 0 & & & & \\
\hline 0 & 487 & & $\begin{array}{l}\text { I y II } \\
\text { III y IV } \\
\text { V }\end{array}$ & $\begin{array}{l}= \\
= \\
=\end{array}$ & $\begin{array}{l}\text { Pliegues transv } \\
\text { Pliegues transv } \\
\text { Atípicos }\end{array}$ & $\begin{array}{l}\text { rersos } \\
\text { jersales }\end{array}$ \\
\hline
\end{tabular}

Cuadro 3

GOMBINAGIONES EN AMBAS MANOS DE PLIEGUES PALMARES TRANSVERSOS, TRANSVERSALES Y CONFIGURACIONES NORMALES EN MAYAS YUCATECOS SERIE MASCULINA $(\mathrm{n}=552)$

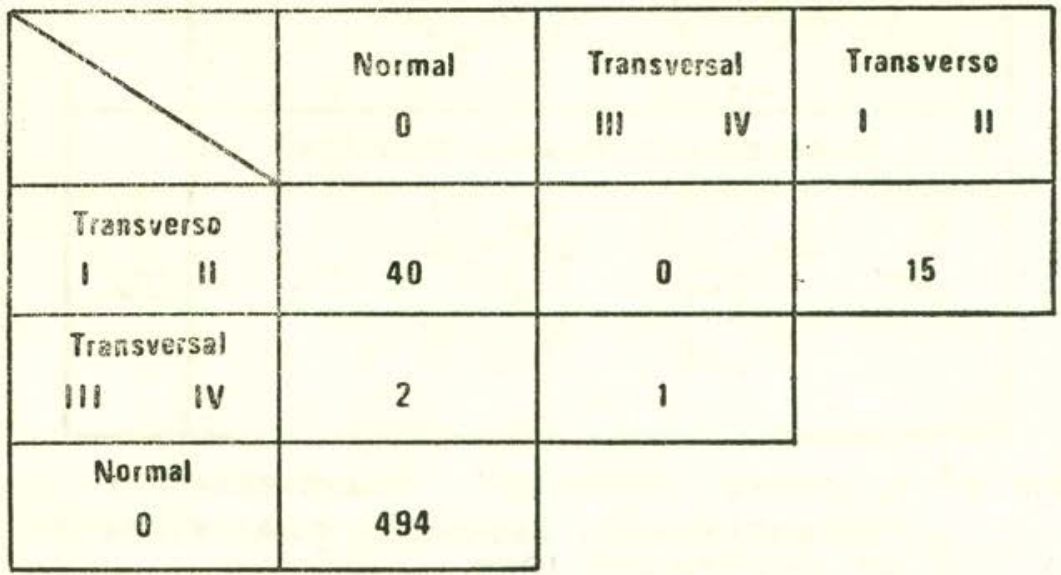




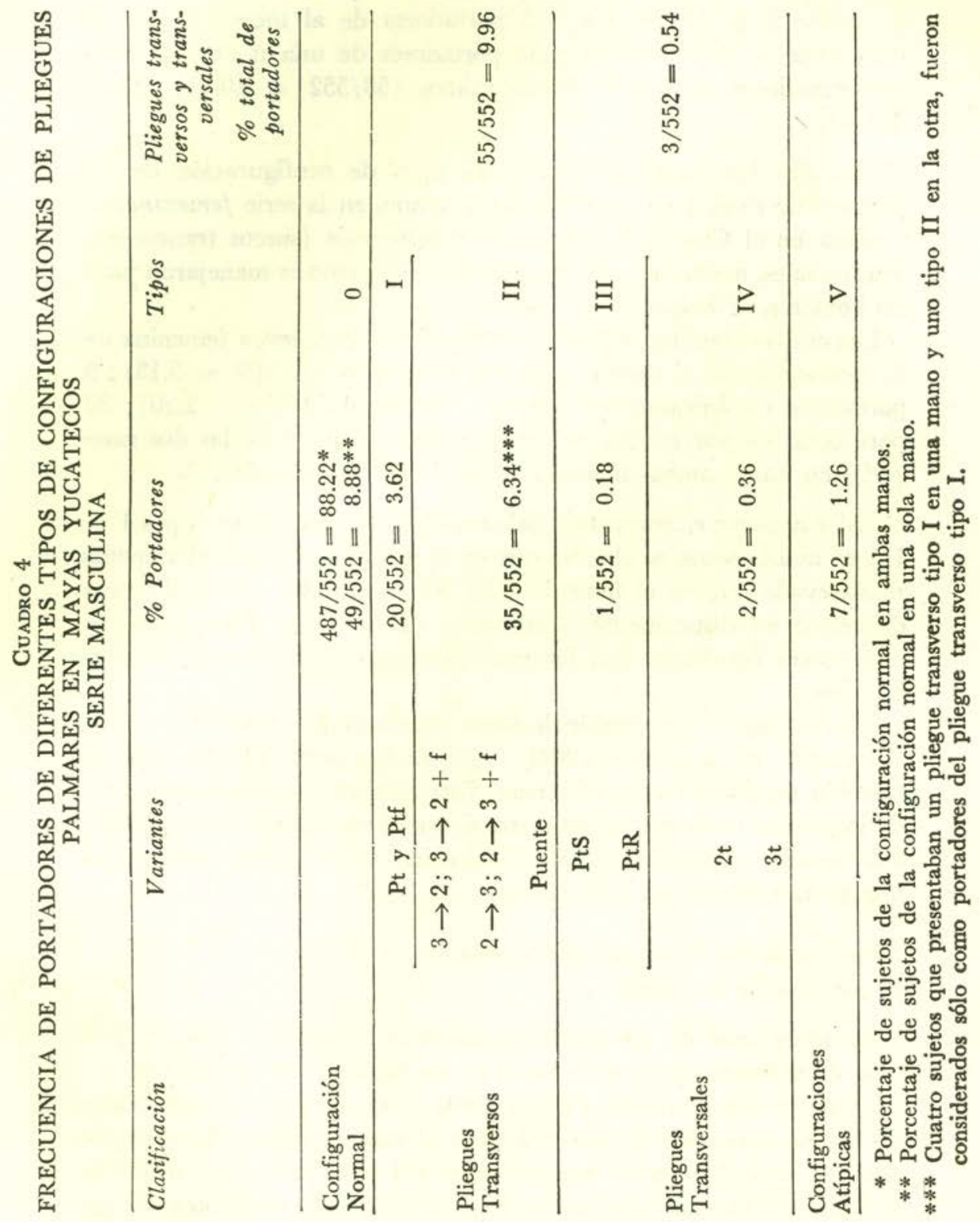

Estudios de Cultura Maya. Vol. XII, 1979

Instituto de Investigaciones Filológicas/

Centro de Estudios Mayas, UNAM 
Para la muestra masculina, integrada por 552 sujetos, se obtuvieron así los resultados siguientes: 55 portadores de por lo menos 1 pliegue transverso $(55 / 552=9.96) ; 3$ portadores de al menos 1 pliegue transversal $(3 / 552=0.54) ; 58$ portadores de una $\mathrm{u}$ otra de tales configuraciones en una o ambas manos $(58 / 552=10.51)$ (Cuadro 4).

b) La distribución de los diferentes tipos de configuración de los pliegues de flexión en la palma de la mano, en la serie femenina, se muestra en el Cuadro 5. Los datos reagrupados (surcos transversos, transversales, normales), de la misma manera como se manejaron para los hombres, se hallan en el Cuadro 6.

Las cifras obtenidas indican la presencia en la muestra femenina de 21 portadoras de al menos 1 pliegue transverso $(21 / 409=5.13) ; 9$ portadoras de únicamente 1 pliegue transversal $(9 / 409=2.20) ; 30$ portadoras de por lo menos 1 pliegue de cualquiera de las dos categorías en una o ambas manos $(30 / 409=7.33$ ) (Cuadro 7).

c) $\mathrm{Al}$ comparar el porcentaje del surco palmar transverso (tipos I + II) en ambos sexos, se observa que en el sexo masculino es claramente más elevado que en el femenino. La $\mathrm{X}^{2}$ es de 7.62, que indica una diferencia estadísticamente significativa $(P>0.01)$. Esta diferencia entre sexos concuerda con los resultados obtenidos en muchos otros estudios.

Sin embargo, el porcentaje de surcos transversales (tipos III + IV), fue mayor en mujeres $(2.20 \%)$ que en hombres $(0.54 \%)$, siendo también significativa la diferencia. Esta distinta incidencia por sexo de los surcos transversales requiere, sin embargo, de una investigación más amplia, aplicando la misma definición de los surcos transversales que se ha utilizado en este trabajo.

\section{Distribución del surco palmar transverso en función de la mano}

La repartición de los pliegues transversos (tipos I + II), en función de la mano, para cada sexo, se encuentra en el Cuadro 8.

Lestrange ha mostrado $(1967,1969)$ que no existe independencia de ambas manos en lo que concierne al surco palmar transverso, es decir, la probabilidad de manifestación del carácter en una mano no es absolutamente independiente de su manifestación en la otra, ya que existe un número apreciable de sujetos que presentan el carácter en ambas manos. Por ello, si se desea comparar la incidencia del pliegue transverso en función de la mano, debe tomarse sólo en consideración a los individuos portadores del pliegue en una sola mano. 
Guadro 5

COMBINACIONES EN AMBAS MANOS DE LOS DIFERENTES TIPOS DE PLIEGUES PALMARES EN MAYAS YUCATECOS SERIE FEMENINA $(n=409)$

\begin{tabular}{|c|c|c|c|c|c|c|}
\hline & 0 & $v$ & IV & Iil & II & 1 \\
\hline 1 & 3 & 0 & 0 & 0 & 0 & 2 \\
\hline 11 & 16 & 0 & 0 & 0 & 0 & \\
\hline III & 2 & 0 & 0 & 0 & & \\
\hline IV & 7 & 0 & 0 & & & \\
\hline$v$ & 5 & 0 & 0 & $=$ & Configuración & normal \\
\hline 0 & 374 & & $\begin{array}{l}\text { I y II } \\
\text { III y IV } \\
\text { V }\end{array}$ & $\begin{array}{l}= \\
= \\
=\end{array}$ & $\begin{array}{l}\text { Pliegues transv } \\
\text { Pliegues transv } \\
\text { Atípicos }\end{array}$ & $\begin{array}{l}\text { versos } \\
\text { versales }\end{array}$ \\
\hline
\end{tabular}

Cundro 6

COMBINACIONES EN AMBAS MANOS DE PLIEGUES PALMARES TRANSVERSOS, TRANSVERSALES Y CONFIGURACIONES

NORMALES EN MAYAS YUGATECOS

SERIE FEMENINA $(n=409)$

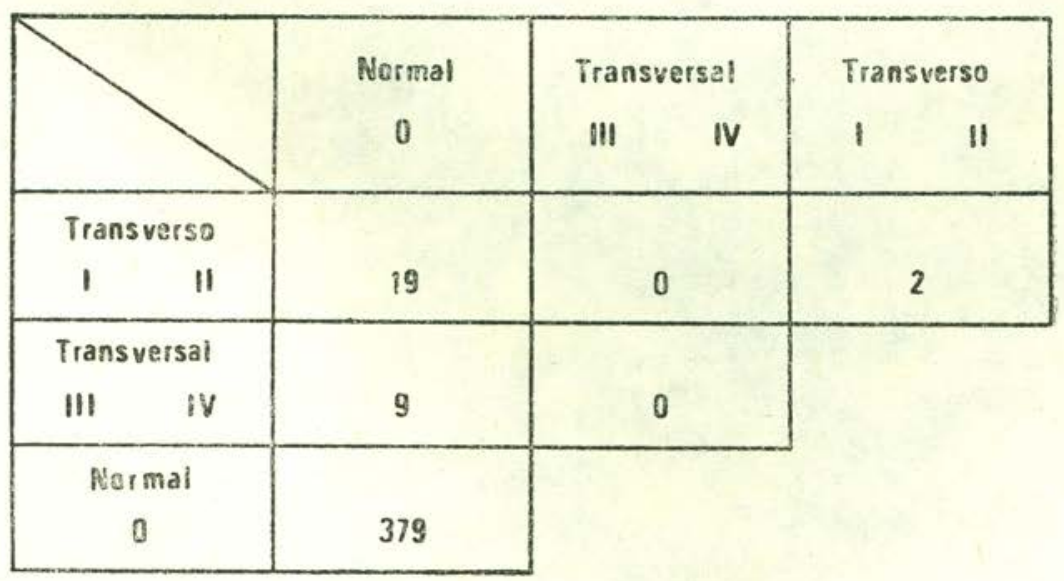




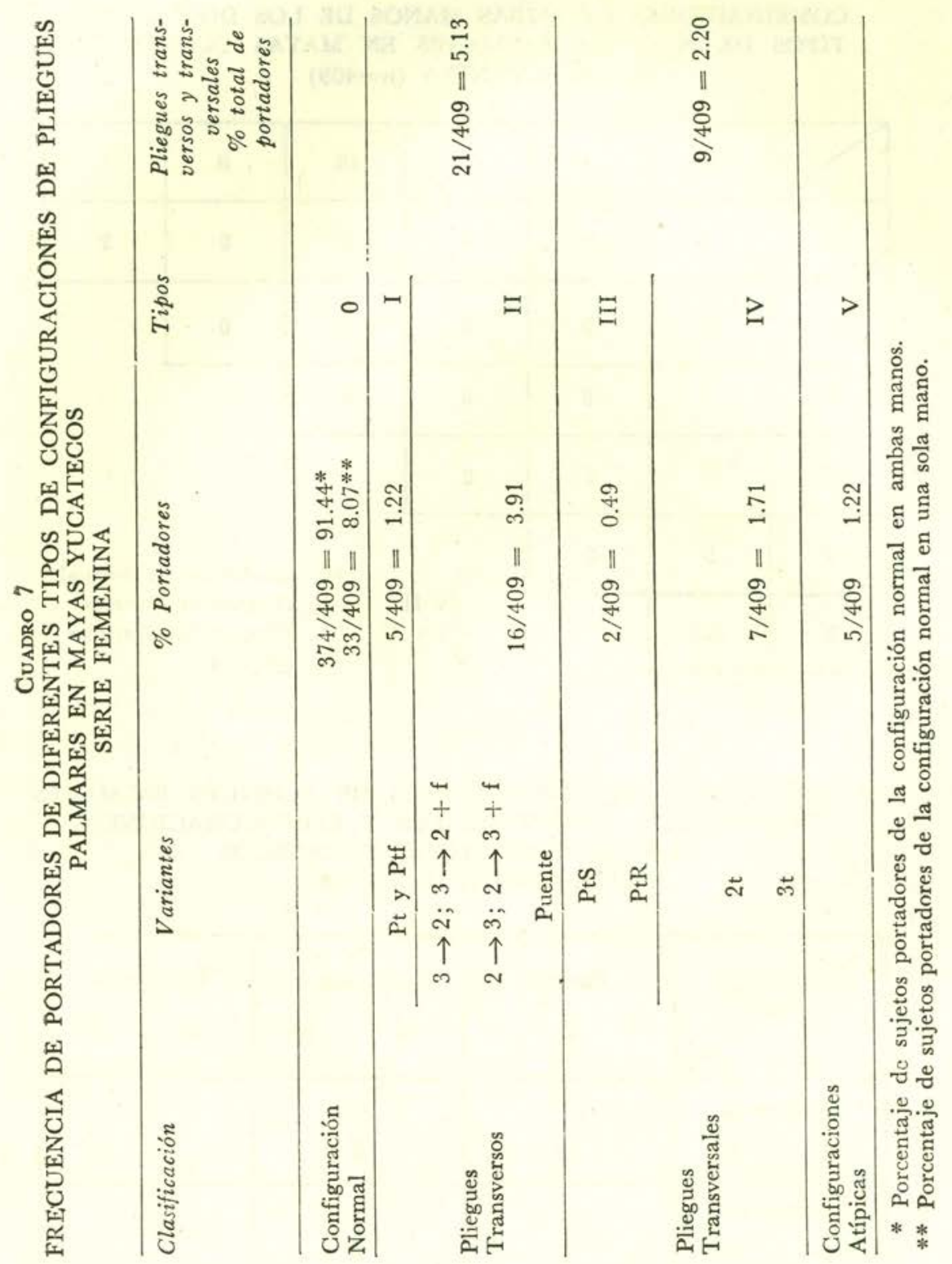

Estudios de Cultura Maya. Vol. XII, 1979

Instituto de Investigaciones Filológicas/

Centro de Estudios Mayas, UNAM 
En la muestra maya estudiada, en efecto, se observa en la serie masculina que de un total de 55 portadores del pliegue transverso, un porcentaje importante lo presentaba en ambas manos $(15 / 55=\ldots$ $37.5 \%$ ), si bien en la serie femenina, el porcentaje de pliegue transverso bilateral -en relación al total de portadoras- baja a sólo $10.53 \%$ ( $2 / 23$ casos).

Cuadro 8

DISTRIBUCION DEL PLIEGUE TRANSVERSO

(TIPOS I + II) EN FUNCION DE LA MANO, EN MAYAS YUCATECOS

\begin{tabular}{lcccccc}
\hline Sexo & $\begin{array}{c}\text { Total } \\
\text { de } \\
\text { sujetos }\end{array}$ & $\begin{array}{c}\text { No. total } \\
\text { de porta- } \\
\text { dores } y \%\end{array}$ & $\begin{array}{c}\text { Sólo en } \\
\text { mano } \\
\text { derecha }\end{array}$ & $\begin{array}{c}\text { Sólo en } \\
\text { mano iz- En ambas } \\
\text { quierda }\end{array}$ & $\begin{array}{c}\text { ananos } \\
\text { manosy }\end{array}$ \\
\hline Hombres & 552 & 55 & 9.96 & 21 & 19 & 15 \\
Mujeres & 409 & 21 & 5.13 & 10 & 9 & 2 \\
\hline
\end{tabular}

Ahora bien, si se tiene en cuenta la incidencia unilateral del surco transverso, sea en la mano izquierda o en la derecha, se puede observar que en nuestras muestras de ambos sexos las diferencias constatadas son insignificantes.

Este resultado contraría la observación realizada en la mayoría de las series estudiadas de una frecuencia más elevada en la mano izquierda que en la derecha, diferencia que se acentúa más en las mujeres que en los hombres. Sólo nuevos estudios y muestras suficientemente numerosas, podrían precisar una diferencia real — si la hay- de la incidencia por mano del surco palmar transverso.

El surco palmar transverso en los mayas, grupos asiáticos y otros aborígenes americanos

El estudio comparativo del carácter que hemos venido examinando, considerando los datos consignados en la literatura, presenta limitaciones de orden metodológico, además de la ya comentada escasez de información publicada para pueblos de la zona asiática y americana.

Así, las diferentes nomenclaturas que han sido utilizadas por los investigadores y la inadecuada definición de éstas, que permitan apreciar su grado de concordancia, impiden establecer en muchos casos comparaciones confiables. La forma de presentación de los resultados, si se consideran incidencias por número de manos y no por portadores del carácter, o si se presentan únicamente los datos reunidos para ambos sexos, imposibilitan en otras ocasiones la utilización comparativa de tales datos. 


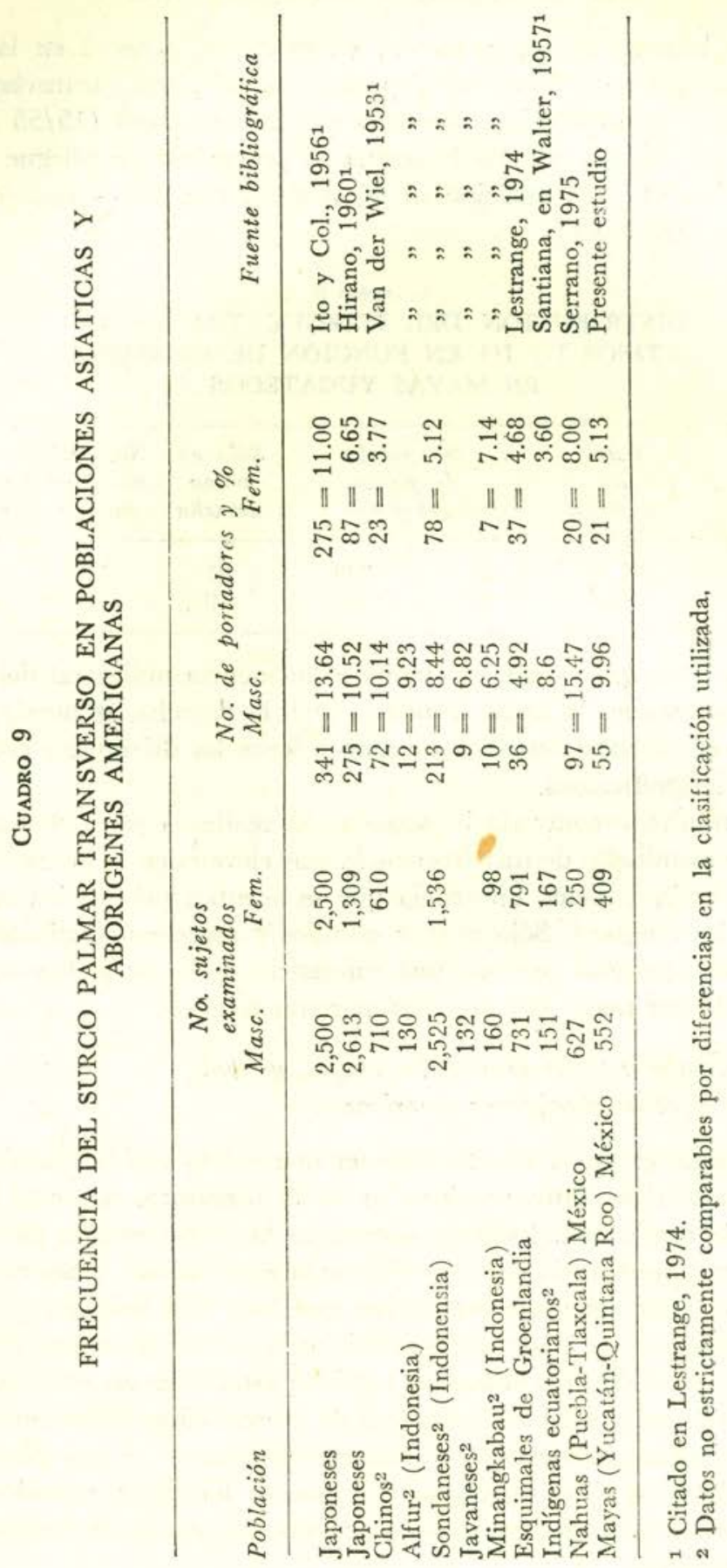


No obstante estas limitaciones, trataremos de situar comparativamente los resultados obtenidos en los mayas yucatecos, en relación a otros grupos indígenas americanos y asiáticos. Para ello, nos basaremos en los datos recopilados por Lestrange (1974) en su estudio sobre los esquimales de la costa oriental de Groenlandia, en el cual reúne críticamente las informaciones sobre pliegue transverso en diversas poblaciones de filiación asiática.

En el Cuadro 9 se presentan los datos referentes a diversos grupos humanos, indicando aquellos casos en que las muestras no son estrictamente comparables, en virtud de la falta de precisión de la forma como se definió el carácter en estudio o el criterio con que se elaboraron los datos (número de manos o de portadores del carácter).

Tales datos, pueden proporcionar una visión general de la incidencia del pliegue transverso en las poblaciones que ahora nos interesan. Se han omitido del Cuadro 9 solamente algunas muestras muy pequeñas, que por esta razón incrementan la reserva con que deben tomarse los datos respectivos, teniendo en cuenta que la reducida frecuencia del carácter estudiado precisa para resultados confiables de muestras muy numerosas.

Con tales reservas, no obstante, a partir de los datos consignados en el Cuadro 9, podemos considerar con Lestrange (1974, p. 120), que en las poblaciones xantodermas o mongoloides, la frecuencia del pliegue transverso es de aproximadamente $10 \%$ en los hombres y $6.5 \%$. en las mujeres. Se trata de frecuencias superiores a las que presentan los europeos, alrededor de $5 \%$ en hombres y $2.5 \%$ en mujeres.

En los grupos americanos se presentan variaciones considerables, aun cuando para apreciarlas no contemos más que con las muy escasas muestras antes consignadas. Así, los esquimales presentan frecuencias muy bajas de surco palmar transverso, lo cual los distingue netamente del resto de grupos de filiación mongoloide.

En cuanto al grupo maya que hemos estudiado, se ubica perfectamente en la gama de variación de frecuencias del pliegue transverso en pueblos mongoloides; las cifras obtenidas son muy parecidas a las reportadas para algunas series asiáticas como la de japoneses de Hirano (1960).

Sin embargo, podemos observar que existen diferencias con los otros dos grupos amerindios enlistados (indígenas ecuatorianos y nahuas del centro de México). Si retenemos nuestra atención en esta última serie, enteramente comparables puesto que se aplicó en su estudio una metodología equivalente, encontramos que la frecuencia del pliegue transverso es bastante más alta que en nuestra serie maya, situándose 
incluso en el margen superior de la variación constatada para poblaciones mongoloides.

Se trata de dos grupos mesoamericanos que comparten en gran medida una misma tradición histórica y cultural, pero presentan contrastes somatológicos evidentes, en los cuales se incluye el carácter que examinamos; se suscita así el problema de sus relaciones biológicas más precisas y sus afinidades genético-antropológicas básicas.

En esta perspectiva, por el momento, no queda más que señalar la situación precaria del estudio bioantropológico de los grupos indígenas americanos y la necesidad de conjuntar informaciones más amplias, como la que hemos querido aportar en este trabajo, que permitan eventualmente la aclaración de problemas sobre el incesante cambio biocultural y sobre los nexos antropológicos de los grupos indígenas que, como el maya, despiertan numerosas interrogantes a la investigación.

\section{Resumen}

Se ha examinado la frecuencia del surco palmar transverso en un grupo de población maya-yucateca (552 hombres y 409 mujeres), en cuanto a su manifestación por sexo y por lado y la incidencia general del carácter en este grupo, comparativamente a otras poblaciones asiáticas y amerindias.

Se constató, tal como en otros estudios similares, una frecuencia más elevada del carácter en la serie masculina. Sin embargo, la distribución por lado - mano derecha, mano izquierda - en ambos sexos fue sensiblemente la misma, a diferencia de los resultados de otros estudios que señalan una frecuencia mayor en la mano izquierda, sobre todo en la serie femenina.

La frecuencia del surco palmar transverso típico fue de 3.62 en hombres y 1.22 en mujeres, cifras que pueden considerarse bajas, aunque se incrementan notablemente si se consideran las formas transicionales, que al parecer tienen una misma etiología. En este sentido, la frecuencia del pliegue transverso alcanza $9.96 \%$ en el sexo masculino y $5.10 \%$ en el femenino, que cae en el margen de variación de las poblaciones de filiación mongoloide.

En los grupos indígenas americanos, a juzgar por las pocas series estudiadas, existen aparentemente diferencias de la frecuencia del surco palmar transverso. Los mayas presentan las cifras más bajas reportadas hasta ahora (exceptuando a los esquimales que en rigor no se clasifican como amerindios). No obstante, se requieren nuevos 
trabajos en el contexto de la investigaciones somatológica de las poblaciones amerindias, que permitan esclarecer sus inter-relaciones biológicas y sus particularidades étnicas dentro del tronco racial mongoloide.

\section{BIBLIOGRAFIA}

Almeida Santos, M. S. DE

1960 O sulco palmar transverso nos portugueses. Contribuições para o.

BRANDER, T. Estudo da Antropologia Portuguesa, Vol. 7, facs. 3o., pp. 41-44.

1940 Uber mongoloide partial Symptome mit besonderer Beachtung der sogenaunten Vierfingerfurche. Acta Paidiat. Uppsala, 28, suppl. 1, pp. 21-38.

Hirano, I.

1960 Untersuchungen über die Vierfinger oder Affenfurche bei Japanern (en Japonés). Niigataigakkai Zassi, 74, pp. 1526-1549.

HoLt, S.

1968 The Genetics of Dermal Ridges. Charles C. Thomas Publisher, Springfield Illinois, U. S. A.

Ito, M. Ogata, T. Murai M., y Osugi, $H$.

1956 Die einteilung sowie entstehungsverhaeltnisse der affen furche bei den Japanern (en Japonés). Revista japonesa.

Kherumian R., Moulleg J., Durand M., Metianu C., Desabie J. y Kherumian Allary, O.

1955 Contribution à l'étiologie génétique des cardiopathies congénitales: le pli palmaire transverse. Anthropologie différentielle et

Kimura, K. Science des types constitutionnels, No. 3, pp. 333-342, Geneve.

1968 A study of palmar crease in Ainu. Zinruigaku Zassi. The Journal of Anthropological Society of Nippon. Vol. 76, No. 759, pp. 60-74.

Lestrange, M. Th. DE

1967 Le pli transverse: nouvelle étude de sa répartition chez les habitants de Plozévet (Finistére Sud, France). Cahiers du C. R. A., No. 8, en: Bull. et Mém. Soc. d'Anthrop. de Paris, Vol. 2, XIIe série, pp. 183-191.

1969 A propos des plis de flexion de la paume: Classification et rapports entre les différents types décrits. Cahiers du C.R. A., Nos. 9 y 10, en: Bull. et Mém. de la Soc. d'Anthrop. de Paris, Vol. 5, XIIe série, pp. 251-267.

1974 Le pli Transverse Chez les Eskimo D'Amassalik (Côte est du Groenland). Comparaison avec les données concernant d'autres populations xanthodermes. Cahier du C. R. A. No. 13, Supplémentà Bull. et Mém. de la Soc. d'Anthrop. de Paris. Vol. 2, 13e série, pp. 109-122. 
Schaumann, B. y M. Alter

1975 Dermatoglyphics in Medical Disorders. Springer-Verlag. New York.

VIlla Rojas

1945 The Maya of East Central Quintana Roo. Carnegie Institution of Washington. Washington, D. C.

Weninger, M. y L. Navratil

1957 Die Vierfingerfuche in ätiologischer Betrachtung. Anthrop. Gesselsch. in Wien, Bd. LXXXVII. 